\title{
1 An Oral Delivery System for Controlling White Spot Syndrome Virus Infection in Shrimp
}

\section{Using Transgenic Microalgae}

3 Asama Kiataramgul ${ }^{1}$, Sugunya Maeenin ${ }^{1}$, Saul Purton ${ }^{2}$, Ikuo Hirono ${ }^{3}$, Thanyanan Wannathong ${ }^{3, *, \#}$ and

4 Sasimanas Unajak ${ }^{4, *, \#}$

5

$6{ }^{1}$ Department of Biochemistry, Faculty of Science, Kasetsart University, 50 Ngam Wong Wan Road, Chatuchak, 7 Bangkok, Thailand, 10900

$8 \quad{ }^{2}$ Algal Research Group, Institute of Structural and Molecular Biology, University College London, Gower

9 Street, London, WC1E 6BT, United Kingdom

$10{ }^{3}$ Graduate School of Marine Science and Technology, Tokyo University of Marine Science and Technology,

11 Konan 4-5-7, Minato-Ku, Tokyo 108-8477, Japan.

$12{ }^{4}$ Department of Biology, Faculty of Science, Silpakorn University, 6 Rajamankha Nai Road, Amphoe Muang,

13 Nakhon Pathom, Thailand, 73000

*Corresponding author

$18 \quad{ }^{\ddagger}$ These authors contributed equally to this study

19 Assist Prof. Sasimanas Unajak, Ph.D.

Department of Biochemistry, Faculty of Science, Kasetsart University,

2150 Ngam Wong Wan Road, Chatuchak, Bangkok 10900

Tel: +66 (2) 562 5555, Fax: + 66(2) 561-4627

Email: fscissmn@ku.ac.th (S. Unajak)

Thanyanan Wannathong Brocklehurst, Ph.D.

Department of Biology, Faculty of Science, Silpakorn University, 6 Rajamankha Nai Road, Amphoe Muang,

27 Nakhon Pathom 73000

Tel: +66 (34) 245327, Fax: +66 (34) 245325 
ABSTRACT

32

White spot disease (WSD) is a longstanding and serious viral disease of various shrimp species that has caused high mortality rates for many decades. Currently, there is no practical method to control this disease. Therefore, we have explored the development of a novel vaccine-based method to control this disease using transgenic algae. During infection by white spot syndrome virus (WSSV), the interaction between viral envelope proteins and cell surface protein receptors on target cells is the key step of viral entry and replication. Hence, transgenic lines of the green microalga Chlamydomonas reinhardtii harboring a WSSV VP28 viral envelope protein were created as an oral delivery system for vaccinating shrimp. Two type of transplastomic lines containing wild-type and codon optimized gene sequences for VP28 were evaluated for recombinant protein levels. Only the codon optimized line gave rise to detectable VP28 in western blot analysis, which demonstrated that optimization for chloroplast codon bias improved the efficiency of expression and that the gene design produced a favorable RNA secondary structure with suitable free energy for translation. In addition, bile salt and acid tolerance tests demonstrated this transgenic Chlamydomonas can tolerate mildly acidic ( $\mathrm{pH} 4.0)$ conditions and $0.30 \%$ bile salts. These features indicated that algal cells are suitable for delivering viral antigen through a shrimp's digestive system. In WSSV infection experiments, the highest survival rate (87\%) was recorded in shrimps fed with the codon optimized VP28 line mixed into their feed indicating that this line could be employed in the control of WSSV spread in shrimp populations. This algal strategy offers an alternative new, efficient, fast and less labor-intensive method for the control of other diseases in aquatic animals through oral delivery.

Keywords: White spot syndrome virus; oral delivery system; Chlamydomonas reinhardtii; chloroplast genetic engineering; transgenic algae 
59

60

61

62

63

64

65

66

67

68
- An oral delivery system to control WSSV disease in shrimp has been developed based on an edible microalga engineered to synthesize a viral antigen in the chloroplast.

- A codon optimized synthetic gene encoding VP28 protein from WSSV was successfully integrated into the chloroplast genome of $C$. reinhardtii.

- Oral administering of the transgenic alga effectively raised the survival rate of shrimp exposed to WSSV compared to a control group.

- The cell wall deficient $C$. reinhardtii strain used is suited as a shrimp supplement as it can persist for 80 minutes in conditions of mild acid (pH 4.0$)$ and $0.15 \%$ bile salts similar to that found in the shrimp digestive system. 
White spot disease (WSD) is an infectious disease of decapod crustaceans such as shrimp, lobster and crayfish and is caused by white spot syndrome virus (WSSV) [1]. The virus particularly affects farmed shrimp such as Litopenaeus vannamei (whiteleg shrimp) and Penaeus monodon (Asian tiger shrimp) and is a global problem for the shrimp industry because practical WSD prevention and control methods during cultivation are poorly developed. Most of the methods rely on the application of different effective biomolecules such as purified protein antigens or antibodies, DNA or RNA vaccines, or natural products [2]. However, a practical method for administration of these biomolecules is required that does not adversely impact shrimp health through handling of individuals; is able to be administered to shrimp of all growth stages; is suitable for treating large numbers of animals, and is time-saving and does not require skilled personnel. Thus, from those requirements, the oral administration of the biomolecule as a supplement in the shrimp feed is an attractive alternative to direct delivery. VP28, an envelope protein of WSSV, is recognized as a key target for controlling WSD since the protein plays a central role in the infection process through its interaction with PmRab7, a shrimp cell surface protein [3]. Various control methods using VP28 have been evaluated, such as DNA vaccines [4, 5], RNA interference and double-stranded RNA $[6,7,8]$ and use of recombinant VP28 (rVP28) [9 - 13]. However, most of these methods employ injection into shrimp as the delivery system, which is not practical or cost-effective. Therefore, various oral delivery systems have been developed and have shown promising disease control. For example, using attenuated bacteria carrying VP28 DNA [14], baculovirus with VP28 expressed on the surface [15], biomaterials such as chitosan as a DNA-nanoparticle complex [16] and double-stranded RNA or protein vaccines synthesized in transgenic alga $[7,17,18]$. However, the major concern of an oral administration method is compatibility of the delivery system and the gastrointestinal tract of the recipient animal. Therefore, a practical oral delivery system should allow the bioactive molecule to persist in the acidic and bile salt environments and then be released at a suitable position in the gastrointestinal tract.

The unicellular microalga, Chlamydomonas reinhardtii offers significant potential as a light-driven cell factory for synthesis of recombinant proteins such as subunit vaccines [19 - 22]. Several aspects in particular are attractive for the development of oral vaccines. Firstly, transgenes can be stably integrated into the chloroplast genome at specific loci as a result of efficient homologous recombination, and high-level expression of the transgenes can be achieved [23]. Secondly, the availability of both walled and cell-wall deficient $C$. reinhardtii strains allows the tailoring of the breakdown characteristics of the algal cell in the shrimp gastrointestinal tract. 
99 line was shown to be stable under conditions that mimic that in the shrimp gut, and when included in the feed

101 for shrimp immunostimulant/vaccination than other methods such as injection, and the use of the algal platform 102 could be exploited to control other diseases in economically important aquatic animals. 


\section{Algal strains and growth conditions}

Chlamydomonas reinhardtii strains were maintained on tris-acetate phosphate (TAP) medium with $2 \%$

\section{Codon optimization}

The sequence of WSSV envelope protein VP28 was obtained from GenBank (accession number: DQ681069.1). The DNA sequence encoding WSSV including a C-terminal polyhistidine tag was optimized to be compatible with $C$. reinhardtii's chloroplast codon usage (http://www.kazusa.or.jp) by using Codon Usage Optimizer (CUO) beta 0.92 program (http://www.ucl.ac.uk/algae/Genetic_engineering_tools). Multiple sequence alignment and percent similarity were predicted using GeneDoc (Ver. 2.7.000) and ClustalW2 (http://www.ebi.ac.uk/Tools/msa/clustalw2/). Codon bias was verified by computing the expected codon adaptation index (e-CAI). Secondary structure of the optimized VP28 mRNA was predicted using mfold (http://mfold.rna.albany.edu/). The codon optimized DNA for $v p 28$ (termed "vp28-op") was synthesized by Integrated DNA Technology (IDT, USA) and included a unique SapI and SphI site upstream and downstream of the coding region, respectively.

\section{Plasmid construction}

To construct a plasmid carrying the wild type version of $v p 28$ the gene was amplified by PCR from pET-vp28 by using primers CrVP28F: 5'-GCTCTTCAATGGATCTTTCTTTCACTCTTTCGGTC-3' and CrVP28R: 5'-GCATGCCTAGTGATGGTGATGGTGATGCTC, which contained SapI and SphI (underlined) sites. Both $v p 28$ and $v p 28$-op were then cloned into the chloroplast expression vector pASapI [25] at corresponding restriction sites and transformed into E. coli (DH5 $\alpha$ ). Transformed cells were screened by PCR using specific primers for pASapI; pASapF: 5'-CAAGTGATCTTACCACTCAC-3' and pASapR: 5'CAAACTTCACATGCAGCAGC-3'. The nucleotide sequence of $v p 28$ and $v p 28$-op in the two plasmids was 
checked by DNA sequencing (Macrogen, Korea) and the plasmids designated as pASapI- $v p 28$ and pASapIvp28op, respectively.

\section{Transformation of Chlamydomonas reinhardtii}

Chloroplast expression vectors, $\mathrm{pASapI}-\mathrm{vp} 280 \mathrm{p}$, $\mathrm{pASapI}-\mathrm{vp} 28$, and empty $\mathrm{pASapI}$, were introduced to chloroplast of C. reinhardtii by glass bead transformation as described previously in Economou et. al. [26]. A $300 \mu 1$ of mid-log phase (about $2 \times 10^{6}$ cells.mL ${ }^{-1}$ ) TN72 were resuspended with HSM broth and transferred to a sterile tube containing $300 \mathrm{mg}$ glass beads of diameter 425-600 $\mu \mathrm{m}$ (Sigma-Aldrich, U.S.). Five microgram of plasmid was added and vigorously agitated for $15 \mathrm{sec}$. Warmed $0.5 \%$ Bacto $^{\mathrm{TM}}$ Agar (Becton Dickinson, U.S.) was added, quickly poured onto an HSM agar plate and incubated at $23^{\circ} \mathrm{C}$ in $\operatorname{dim} \operatorname{light}\left(1-5 \mu \mathrm{E} \cdot \mathrm{m}^{-2} \cdot \mathrm{s}^{-1}\right)$ overnight, then moved to a higher light $\left(40-50 \mu \mathrm{E} \cdot \mathrm{m}^{-2} . \mathrm{s}^{-1}\right)$ for 2-4 weeks. Putative algal transformant colonies were restreaked onto fresh HSM agar plates, cultured under light intensity of $40-50 \mu \mathrm{E} \cdot \mathrm{m}^{-2} \cdot \mathrm{s}^{-1}$ at $23^{\circ} \mathrm{C}$ and subcultured for at least three rounds to obtain homoplasmic lines. Subsequently, the obtained phototrophic transformants were verified for transgene integration by PCR using a set of three primers: trnE2-F (5'GTCATTGCGAAAATACTGGTGC-3'), psbH-R (5'-ACGTCCACAGGCGTCGTAAGC-3') and atpA-R (5'GATGACGTTTCTATGAGTTGGG-3') as illustrated in Fig 1. Furthermore, the loss of the aadA gene and restoration of $p s b H$ were confirmed by a 'spot test' growth assay on TAP agar containing $100 \mu \mathrm{g} \cdot \mathrm{mL}^{-1}$ spectinomycin and HSM agar, respectively, under white light for 7 days.

\section{Recombinant protein expression, purification and western blot analysis}

Transgenic algal cells $(100 \mathrm{ml})$ (designated as TNVP28-op, TNVP28 and TN72-emp; C. reinhardtii transformed with pASapI-vp28op, pASapI-vp28, and empty pASapI vector, respectively), were collected and lysed in $50 \mathrm{ml}$ denaturing extraction buffer $(20 \mathrm{mM}$ sodium phosphate buffer $\mathrm{pH} 7.4,500 \mathrm{mM} \mathrm{NaCl}, 8 \mathrm{M}$ urea, $5 \mathrm{mM}$ imidazole and $0.5 \%$ Tween-20). For VP28 expression in E. coli, strain BL21 (DE3) harboring pET-vp28 was induced with $3 \mathrm{mM}$ IPTG and employed as a positive control for western blot analysis. All versions of the VP28 protein were purified using Ni-NTA sepharose 6 Fast Flow (GE Healthcare) under denaturing condition and VP28 eluted by using 100, 250, and $500 \mathrm{mM}$ imidazole. Protein concentration was determined by Bradford assay (Bio-Rad) using BSA as a standard protein.

Proteins separated by $12 \%$ SDS-PAGE were transferred to PVDF membrane (Pall Corporation, USA). Recombinant protein was detected using rabbit polyclonal anti-VP28 (diluted 1:10,000 with TBST) as the 
163 primary antibody (produced in S. Unajak lab). The secondary antibody was anti-rabbit IgG antibody conjugated 164 with horseradish peroxidase (HRP, Cell signaling, USA) and detection used SuperSignal ${ }^{\mathrm{TM}}$ West Pico Chemiluminescent Substrate (Thermo Scientific, UK). The signal was recorded by Chemiluminescence detector (ImagQuant LAS500, GE Health care, USA).

\section{Oral administration and WSSV challenge}

Penaeus vannamei ( at $6-7 \mathrm{~g}$ ) free from WSSV and yellow head virus were divided into five groups (15 shrimps per group and conducted in triplicate) and fed with commercial feed (Sunshine, Thailand) containing recombinant algae (TNVP28-op, TNVP28 and TN72-emp). Control groups (with and without WSSV challenge) were fed with normal feed. Each group was fed twice daily. At 10 days post-stimulation, all groups (except the negative control) were challenged by a cohabitation system with a WSSV-injected moribund shrimp. The mortality rates of the experimental shrimps were recorded daily. This experiment on animals complied with the ethical standards set by the National Research Council of Thailand (NRCT). Statistical analysis of mortality 
relationship was performed with one-way analysis of variance (ANOVA) followed by Duncan's new multiple range test using the SPSS 11.5 program at the significant consideration level of 0.05 .

\section{RESULTS}

\section{Codon optimization of the gene for WSSV VP28}

Chloroplast genes of C. reinhardtii display a strong bias towards AT-rich codons, and several studies have shown that optimization of codon usage in a foreign gene to match this bias can significantly improve the efficiency of translation of the gene in the algal chloroplast [27]. Hence, the VP28 gene was codon-optimized (designated as vp28-op) according to $C$. reinhardtii chloroplast codon usage showing the expected codon adaptation index (eCAI) of 0.987 compared to the CAI of the native VP28 which was 0.642 . The secondary structure analysis of $v p 28-o p$ mRNA revealed a free energy for mRNA folding of $-137.30 \mathrm{kcal}^{\mathrm{mol}}{ }^{-1}$ (Fig 1). A nucleotide sequence comparison of native $v p 28$ and synthetic $v p 28-o p$ showed a $76.42 \%$ similarity without any change in amino acid sequence (Fig 2).

\section{Production of C. reinhardtii transformants harboring WSSV vp28-op or vp28}

Synthetic $v p 28-o p$ and $v p 28$ were cloned into the pASapI vector such that the genes were under the control of the endogenous atpA promoter/5' UTR element, and the 3' UTR from $r b c L$ [25] to create plasmids pASapI-vp28op and pASapI-vp28. These plasmids, together with the empty pASapI vector, were used to transformed the plastome of the algal recipient TN72. Five colonies were obtained from pASapI-vp28op, six from pASapI-vp28 and eight from pASapI-empty and the putative transformant lines were designated as TNVP28-op, TNVP28, and TN72EMP, respectively (data not shown). After three rounds of restreaking under phototrophic growth conditions, the homoplasmicity of the polyploid plastome in the transformants was verified by PCR analysis. Gel electrophoresis of the PCR products showed only a $1.2 \mathrm{~kb}$ band in all transformant lines (Fig 3). This band arises from the transformed copies of the plastome whereas a $1.0 \mathrm{~kb}$ and would arise from untransformed copies. The absence of any detectable $1.0 \mathrm{~kb}$ in the PCR analysis indicates that homoplasmy was successfully obtained in all lines.

Additionally, homologous recombination between sequences on the pASapI plasmids and the TN72 plastome results in the replacement of the $a a d A$ gene conferring spectinomycin with a functional copy of the $p s b H$ gene, allowing photosynthetic growth on minimal medium [25], while the loss of aadA gene results in the creation 
of transformant lines free from an antibiotic-resistance marker (Fig 1). None of the TNVP28-op (PO1-5) and TNVP28 (CV1-6) transformants could survive in TAP containing $100 \mu \mathrm{g} / \mathrm{ml}$ spectinomycin, unlike the untransformed TN72 recipient (Fig 4), further supporting the conclusion that the transgenes had successfully integrated into the plastome at the specific position downstream of $p s b H$ and had replaced the aadA marker.

\section{Determination of protein expression in transgenic Chlamydomonas harboring vp28op or vp28}

To determine whether WSSV VP28 is produced in the transgenic algae, western blot analysis was carried out using a polyclonal rabbit anti-VP28 antibody. The expected size of the protein is $\sim 28 \mathrm{kDa}$ and a distinct band of this size was detected in an E. coli extract expressing vp28 (Fig 5, lane P) and in two transformant lines (OP1 and OP2) containing the codon-optimised $v p 28$. In addition, the antibodies also detect an endogenous protein of $\sim 35 \mathrm{kDa}$ in the algal extracts and this is seen in all extracts including that from the negative control transformant line (CP) and the untransformed strain (TN72). Interestingly, the $28 \mathrm{kDa}$ VP28 band is not detected in the OP line carrying the wild-type (non-optimised) version of $v p 28$, highlighting the benefit of codon optimization for improved synthesis of recombinant protein in the C. reinhardtii chloroplast. His-tag affinity purification of VP28 from OP1 further confirmed that the $28 \mathrm{kDa}$ band detected by the antibodies is the His-tagged VP28 as a subsequent western blot (Fig 6) showed detection of this band in the eluted fractions (E1 - E5) with the $35 \mathrm{kDa}$ band eliminated in the flow-through.

\section{Acid and bile salt tolerance assay}

The efficiency of an oral delivery system is influenced by the effectiveness of digestibility and absorption in the animal's digestive tract. The tolerance of the algal cell to the physical environment of the shrimp gut was determined by assessing the integrity of the cells to changes to acidic conditions and bile salt concentration. The transgenic line TNVP28-op, which carries the cell-wall deficient mutation, was incubated in a range of $\mathrm{pH}$ from 2 to 8 and in various concentrations of bile salt; $0.15 \%, 0.3 \%, 0.6 \%$, and $1.2 \%(\mathrm{v} / \mathrm{v})$, respectively. At $\mathrm{pH} 7$ or in $0.15 \%$ bile salts as control conditions during the analysis, the algal cells showed round bright green coloration and remained intact until the end of the exposure period. In the unfavorable environment of low $\mathrm{pH}$ or high bile salt concentration, the algal cells showed discoloration and extensive cell lysis (Fig. 7 and Fig 8). The lowest concentration of bile salts and $\mathrm{pH}$ that the algal cells remained intact and demonstrated green in color are $0.3 \%$ bile salts and $\mathrm{pH} 5.0$. 


\section{Efficiency of transgenic algae on controlling white spot syndrome}

253 To determine the effectiveness of the transgenic C. reinhardtii harboring VP28 on controlling WSSV 254 infection, Pacific white shrimp were fed with feed mixed with the transgenic line TNVP28-op for 14 days.

255 WSSV-infected shrimp were then added to the aquarium tank allowing co-habitation with the algae-fed shrimp. 256 Shrimp mortality, caused by WSSV infection, started on day 4 of co-habitation with the infected animals. In all 257 the control treatments including shrimp fed the commercial feed without the algal supplement, and those given 258 feed containing the non-expressing algal lines TNVP28 and TN72-emp gradually died within 7 days with a near $2590 \%$ survival rate. However, shrimp fed with TNVP28-op showed the lowest mortality with a relative survival of $26087 \%$, as compared to a $100 \%$ survival for shrimp in an uninfected negative control group (Fig. 9). 
VP28, a dominant structural envelope protein of WSSV, is recognized as an effective biomolecule

263 which can activate shrimp immunity and elicit an anti-viral response [28-32]. VP28 plays as a key role in WSSV

264 infection by specifically binding to host cell receptors such as Rab7 and facilitating viral entry, which further

265 allows viral propagation [33]. Thus, a rationale protection strategy against WSSV infection is dependent on

266 interfering with the interaction between VP28 and the shrimp cell receptor to reduce viral entry and also later on

267 activating a shrimp immunity against the invading pathogen. Consequently, different biological forms of VP28

268 such as DNA vaccine $[4,5,14]$ interfering RNA targeting $v p 28$ expression $[34,35]$ and recombinant VP28

269 protein $[19,36]$ were used and demonstrated promising controlling WSSV infectivity. However, most of these

270 methods rely on injection into individual juvenile animals of the active biomolecule, and this is not considered

271 practical in commercial shrimp production. Moreover, preparation of VP28 either as recombinant protein, or as

272 dsRNA or DNA vaccine are costly and time consuming which is considered a further limitation in the

273 preparation and utilization of these agents in aquaculture.

274 An oral system for delivery of recombinant VP28 protein to shrimp as part of the feeding regime would

275 allow the introduction of this immunostimulant to many animals in a technically simple manner without causing

276 stress or possible injury to the shrimp. Various kinds of biological materials have been employed to encapsulate

277 VP28 such as biodegradable materials (chitosan) [16, 19], bacteria; Bacillus subtilis [37] Escherichia coli [38]

278 and the single cell alga Dunaliella salina [9]. The advantages of encapsulation in expression hosts such as algae

279 or bacteria are that the host not only provides a low-cost platform for synthesis of the recombinant protein, but

280 protects the VP28 in the harsh environment of the shrimp gut until it reaches to the target cells in the

281 gastrointestinal tract. Therefore, one of the most important considerations of a VP28 delivery system is

282 optimizing the release of VP28 at the most suitable site and time after it passes through the shrimp digestive

283 system so that efficient WSSV control can be attained. In this study, oral administration was carried out using a

284 cell-wall deficient microalga expressing VP28 in the chloroplast. The results of the feeding and viral challenge

285 trials show a marked improvement in survival when the shrimp are fed algae containing VP28, indicating that a

286 sufficient amount of the administered protein is surviving digestion and reaching the target cells in the shrimp

287 gut. This promising result suggests that oral administration of transgenic algae is a viable approach to managing 288 viral infection.

289 Several studies have shown that efficient production of a recombinant protein in the C. reinhardtii

290 chloroplast requires codon optimization of the transgene $[18,39,40]$. In this study, VP28 was successfully 
expressed in the algal chloroplast after codon optimization whereas the wild-type (non-optimised) gene from WSSV was poorly expressed such that no VP28 was detectable by western analysis. This improvement might have resulted from a rational nucleotide optimization based on five criterions which are 1) codon usage which resembles that used in alga's chloroplast; 2) an expected codon adaptation index (eCAI) close to $1.0 ; 3$ ) a suitable codon bias with $\mathrm{A}$ and $\mathrm{T}$ nucleotides at the third position rather than $\mathrm{G}$ and $\mathrm{C}[40,41]$; 4) an acceptable folding free energy $\left(\Delta \mathrm{G}=-137.30 \mathrm{kcal} \cdot \mathrm{mol}^{-1}\right)$ [42], and 5) a stable mRNA secondary structure which could play a critical role in the regulation of transcription [43] and translation [44]. In this optimization, the complexity of mRNA secondary structures found in genes with low GC content could increase the folding free energy resulting in the reduction of mRNA stability [45]. Thus, unstable mRNA secondary structure could impair the expression by impairing the binding of ribosome and other trans-acting factors during translational initiation [42].

The ability of algal cells to survive in the shrimp digestive system prior to release of VP28 in the lower tract is one of main features required for an efficient oral delivery system. For shrimp, the duration of feed passing through their digestive system is about $80 \mathrm{~min}$ [47]. Our results demonstrated that after $80 \mathrm{~min}$ of incubation, algal cells could resist mild acid (pH 5.0) and bile salts (0.15\%). Additionally, in the digestive tract, it is not only physiological conditions that affect the release of active contents from algae, but digestive enzymes will also result in cell lysis. The release of sufficient VP28 from the cells to stimulate shrimp immunity therefore reflects a balance between the tolerance of the cells to the conditions in the gut and their active breakdown as part of the normal digestive process.

The shrimp fed with the TNVP28-op strain had a significantly lower mortality rate than the control group and the equivalent TNVP28 strain that had undetectable levels of VP28 (i.e. $\sim 13 \%$ mortality after seven days compared with $\sim 100 \%$ mortality). These results indicate a clear protection from WSSV from the VP28 in the TNVP28-op alga, and that other components in C. reinhardtii dried biomass itself are not responsible for the observed protection against WSSV. Furthermore, the delivery system plays a pivotal role in mediating the protection. Several types of oral VP28 delivery system have been developed using for example B. subtilis spores [48], the marine alga Dunaliella salina [9] and the cyanobacterium Anabaena sp. [49] and different efficacies in controlling WSD were observed. Reported protective effects of crayfish fed with VP28 expressed in D. salina 318 (59\% survival rate), B. subtilis spore (37.9 - 44.8\% survival rate) [48] and Anabaena sp. (68\% survival rate)

319 [49] are lower than those reported using chloroplast engineered Chlamydomonas containing either double stranded RNA (84.1\%) [7] or recombinant VP28 (87\%) [this study]. Even though it is not possible to directly 
321 compare the values obtained in the different studies, the findings do suggest that $C$. reinhardtii represents a good 322 host for expression and oral delivery. Furthermore, C. reinhardtii is considered a harmless feed additive with 323 toxicology studies allowing its classification as a GRAS (Generally Recognised As Safe) organism [50]. In conclusion, transgenic microalga appears to be a good vehicle to deliver immunostimulants in 325 controlling WSD in shrimp through oral administration, and the transgenic C. reinhardtii expressing VP28 326 could be employed to prevent WSSV infection in white shrimp. This delivery system could also offer a new 327 method for preventing other viral diseases in aquatic animals, and represent an environmentally friendly and 328 sustainable system for vaccine production given that the algae are grown by photosynthesis with simple inputs 329 of sunlight, $\mathrm{CO}_{2}$ and basic nutrients. 


\section{ACKNOWLEDMENTS}

331 SU is supported by the Kasetsart University Research and Development Institute (KURDI), Thailand and JSPS-

332 NRCT Asian Core University Program. AK was supported by Thailand Research Fund under the project: The 333 Research and Researchers for Industries Scholarship (RRI) for Masters (Grant number MRG555S009). 
335 No conflict of interest declared.

892

893

894

895

896

897

898

899

900

901

902

903

904

905

906

907

908

909

910

911

912

913

914

915

916

917

918

919

920

921

922

923

924

925

926

927

928

929

930

931

932

933

934

935

936

937

938

939

940

941

942

943

944 
1. Pradeep, B., Rai, P., Mohan, S.A., Shekhar, M.S., Karunasagar, I., 2012. Biology, host range,

2. Feng, S., Wang, C., Hu, S., Wu, Q., Li, A., 2017. Recent progress in the development of white spot syndrome virus vaccines for protecting shrimp against viral infection. Arch Virol. 162 (10), 2923-36.

3. Sritunyalucksana, K., Wannapapho, W., Lo, C. F., Flegel, T. W., 2006. PmRab7 is a VP28binding protein involved in white spot syndrome virus infection in shrimp. J. Virol. 80, 1073410742 .

4. Rout, N., Kumar, S., Jaganmohan, S., Murugan, V., 2007. DNA vaccines encoding viral envelope proteins confer protective immunity against WSSV in black tiger shrimp. Vaccine. 25, 2778-2786.

5. Li, X., Liu, Q.-hui, Huang, L.H., 2010. Effect of VP28 DNA vaccine on white spot syndrome virus in Litopenaeus vannamei. Aquaculture International. 18, 1035-1044.

6. Fei, Z., Zhang, X., 2011. The antiviral vp28-siRNA expressed in bacteria protects shrimp against white spot syndrome virus (WSSV). Aquaculture. 319, 311-314.

7. Charoonnart P., Worakajit N., Zedler J.A.Z., Meetam M., Robinson C., Saksmerprome V., 2019. Generation of microalga Chlamydomonas reinhardtii expressing shrimp antiviral dsRNA without supplementation of antibiotics. Sci Rep. 9(1). https://doi.org/10.1038/s41598-01939539-x.

8. Taju, G., Madan, N., Abdul Majeed, S., Kumar, T.R., Thamizhvanan, S., Otta, S.K., Sahul Hameed, A.S., 2015. Immune responses of whiteleg shrimp, Litopenaeus vannamei (Boone, 1931), to bacterially expressed dsRNA specific to VP28 gene of white spot syndrome virus. J. Fish. Dis. 38, 451-465.

9. Feng, S., Zhao, L., Gu, H., Li, Q., Shi, K., Guo, S., Zhang, N., 2014. Preparation of transgenic Dunaliella salina for immunization against white spot syndrome virus in crayfish. Arch. Virol. 159, 519-525.

10. Ha, Y.M., Soo-Jung, G., Thi-Hoai, N., Ra, C.H., Kim, K.H., Nam, Y.K., Kim, S.K., 2008. Vaccination of shrimp (Penaeus chinensis) against white spot syndrome virus (WSSV). J. Microbiol. Biotechnol. 18, 964-967.

11. Thomas, A., Sudheer, N.S., Viswanathan, K., Kiron, V., Bright Singh, I.S., Narayanan, R.B., 2014. Immunogenicity and protective efficacy of a major White Spot Syndrome Virus (WSSV) envelope protein VP24 expressed in Escherichia coli against WSSV. J. Invertebr. Pathol. 123, $17-24$. 
12. Valdez, A., Yepiz-Plascencia, G., Ricca, E., Olmos, J., 2014. First Litopenaeus vannamei WSSV $100 \%$ oral vaccination protection using CotC: $\mathrm{Vp} 26$ fusion protein displayed on Bacillus subtilis spores surface. J. Appl. Microbiol. 117, 347-357.

13. Solís-Lucero, G., Manoutcharian, K., Hernández-López, J., Ascencio, F., 2016. Injected phagedisplayed-VP28 vaccine reduces shrimp Litopenaeus vannamei mortality by white spot syndrome virus infection. Fish Shellfish Immunol. 55, 401-406.

14. Ning, J.F., Zhu, W., Xu, J.P., Zheng, C.Y., Meng, X.L., 2009. Oral delivery of DNA vaccine encoding VP28 against white spot syndrome virus in crayfish by attenuated Salmonella typhimurium. Vaccine. 27, 1127-1135.

15. Syed, M.S., Kwang, J., 2011. Oral vaccination of baculovirus-expressed VP28 displays enhanced protection against White Spot Syndrome Virus in Penaeus monodon. PLoS One. 6, e26427. https://doi.org/10.1371/journal.pone.0026428.

16. Rajeshkumar, S., Venkatesan, C., Sarathi, M., Sarathbabu, V., Thomas, J., Anver Basha, K., Sahul Hameed, A.S., 2009. Oral delivery of DNA construct using chitosan nanoparticles to protect the shrimp from white spot syndrome virus (WSSV). Fish Shellfish Immunol. 26. https://scite.ai/reports/10.1016/j.fsi.2009.01.003.

17. Somchai P, Jitrakorn S, Thitamadee S, Meetam M, Saksmerprome V., 2016. Use of microalgae Chlamydomonas reinhardtii for production of double-stranded RNA against shrimp virus. Aquac Reports. 3, 178-183.

18. Feng, S., Feng, W., Zhao, L., Gu, H., Li, Q., Shi, K., 2014. Preparation of transgenic Dunaliella salina for immunization against white spot syndrome virus in crayfish. Arch Virol. 159 (3), 519 25 .

19. Dyo, Y.M., Purton, S., 2018. The algal chloroplast as a synthetic biology platform for production of therapeutic proteins. Microbiol (United Kingdom). 164 (2), 113-21.

20. Scaife, M.A., Nguyen GTDT, Rico, J., Lambert, D., Helliwell, K.E., Smith, A.G., 2015. Establishing Chlamydomonas reinhardtii as an industrial biotechnology host. Plant J. 82 (3), $532-546$.

21. Charoonnart, P., Purton, S., Saksmerprome, V., 2018. Applications of microalgal biotechnology for disease control in aquaculture. Biology (Basel). 7(2). https://doi.org/10.3390/biology7020024

22. Schroda, M., 2019. Good News for Nuclear Transgene Expression in Chlamydomonas. Cells. 8 (12). https://doi.org/10.3390/cells8121534.

23. Taunt, H.N., Stoffels, L., Purton, S., 2018. Green biologics: The algal chloroplast as a platform for making biopharmaceuticals. Bioengineered. 9 (1), 48-54.

24. Harris, E.H., Stern, D.B., Witman, G.B., 2009. The Chlamydomonas Sourcebook; Durham, North Carolina, USA; London: Academic Press. Vol. 3, pp 2000. 
25. Wannathong, T., Waterhouse, J.C., Young, R.E.B., Economou, C.K., Purton, S., 2016. New tools for chloroplast genetic engineering allow the synthesis of human growth hormone in the green alga Chlamydomonas reinhardtii. Appl Microbiol Biotechnol. 100 (12), 5467-77.

26. Economou, C., Wannathong, T., Szaub, J., Purton, S., 2014. A simple, low-cost method for chloroplast transformation of the green alga Chlamydomonas reinhardtii. Methods Mol Biol. $1132,401-11$.

27. Purton, S., Szaub, J.B., Wannathong, T., Young, R., Economou, C.K., 2013. Genetic engineering of algal chloroplasts: Progress and prospects. Russ J Plant Physiol. 60 (4), 491-9.

28. Witteveldt, J., Vlak, J.M., van Hulten, M.C., 2004. Protection of Penaeus monodon against white spot syndrome virus using a WSSV subunit vaccine. Fish Shellfish Immunol. 16, 571-579.

29. Fu, L.L., Li, W.F., Du, H.H., Dai, W., Xu, Z.R., 2008. Oral vaccination with envelope protein VP28 against white spot syndrome virus in Procambarus clarkii using Bacillus subtilis as delivery vehicles. Lett. Appl. Microbiol. 46, 581-586.

30. Sarathi, M., Simon, M.C., Venkatesan, C., Hameed, A.S., 2008. Oral administration of bacterially expressed VP28dsRNA to protect Penaeus monodon from white spot syndrome virus. Mar. Biotechnol. (NY). 10, 242-249.

31. Witteveldt, J., Vlak, J.M., van Hulten, M.C., 2006. Increased tolerance of Litopenaeus vannamei to white spot syndrome virus (WSSV) infection after oral application of the viral envelope protein VP28. Dis. Aquat. Organ. 70, 167-170.

32. Witteveldt, J., Cifuentes, C.C., Vlak, J.M., van Hulten, M.C.W., 2004. Protection of Penaeus monodon against White Spot Syndrome Virus by Oral Vaccination. J. Virol. 78, 2057-2061.

33. Yi, G., Wang, Z., Qi, Y., Yao, L., Qian, J., Hu, L., 2004. Vp28 of shrimp white spot syndrome virus is involved in the attachment and penetration into shrimp cells. J. Biochem. Mol. Biol. 37, 726-734.

34. Akhila, D.S., Mani, M.K., Rai, P., Condon, K., Owens, L., Karunasagar, I., 2015. Antisense RNA mediated protection from white spot syndrome virus (WSSV) infection in Pacific white shrimp Litopenaeus vannamei. Aquaculture. 435, 306-309.

35. Nilsen, P., Karlsen, M., Sritunyalucksana, K., Thitamadee, S., 2017. White spot syndrome virus VP28 specific double-stranded RNA provides protection through a highly focused siRNA population. Sci. Rep. 7 (1), 1028. https://doi.org/10.1038/s41598-017-01181-w

36. Taengchaiyaphum, S., Nakayama, H., Srisala, J., Khiev, R., Aldama-Cano, D.J., Thitamadee, S., Sritunyalucksana, K., 2017. Vaccination with multimeric recombinant VP28 induces high protection against white spot syndrome virus in shrimp. Dev. Comp. Immunol. 6, 56-64.

37. Fu, L.L., Wanga, Y., Wu, Z.C., Li, W.F., 2011. In vivo assessment for oral delivery of Bacillus subtilis harboring a viral protein (VP28) against white spot syndrome virus in Litopenaeus vannamei. Aquaculture. 322-323, 33-38. 
38. Jha, R.K., Xu, Z.R., Shen, J., Bai, S.J., Sun, J.Y., Li, W.F., 2006. The efficacy of recombinant vaccines against white spot syndrome virus in Procambarus clarkii. Immunol. Lett. 105, 68-76.

39. Franklin, S., Ngo, B., Efuet, E., Mayfield, S.P., 2002. Development of a GFP reporter gene for Chlamydomonas reinhardtii chloroplast. Plant. J. 30, 733-744.

40. Surzycki, R., Greenham, K., Kitayama, K., Dibal, F., Wagner, R., Rochaix, J.D., 2009. Factors effecting expression of vaccines in microalgae. Biologicals. 37 (3), 133-8.

41. Franklin, S.E., Mayfield, S.P., 2004. Prospects for molecular farming in the green alga Chlamydomonas. Curr. Opin. Plant. Biol. 7, 159-165.

42. Kozak, M., 1986. Influences of mRNA secondary structure on initiation by eukaryotic ribosomes. Proc. Natl. Acad. Sci. U S A. 83, 2850-2854.

43. Weik, M., Modrof, J., Klenk, H.D., Becker, S., Mühlberger, E., 2002. Ebola virus VP30mediated transcription is regulated by RNA secondary structure formation. J Virol. 76, 85328539 .

44. Schmittgen, T.D., Danenberg, K.D., Horikoshi, T., Lenz, H.J., Danenberg, P.V., 1994. Effect of 5-fluoro- and 5-bromouracil substitution on the translation of human thymidylate synthase mRNA. J. Biol. Chem. 269, 16269-16275.

45. Jia, M., Li, Y., 2005. The relationship among gene expression, folding free energy and codon usage bias in Escherichia coli. FEBS. Lett. 579, 5333-5337.

46. Gangl, D., Zedler, J.A., Włodarczyk, A., Jensen, P.E., Purton, S., Robinson, C., 2015. Expression and membrane-targeting of an active plant cytochrome $\mathrm{P} 450$ in the chloroplast of the green alga Chlamydomonas reinhardtii. Phytochemistry. 110, 22-28.

47. Beseres, J.J, Lawrence, A.L., Feller, R.J., 2006. Practical equivalence of laboratory and field measurements of gut passage time in two penaeid shrimp species. Mar. Ecol. Prog. Ser. 309, 221231.

48. Ning, D., Leng, X., Li, Q., Xu, W., 2011. Surface-displayed VP28 on Bacillus subtilis spores induce protection against white spot syndrome virus in crayfish by oral administration. J. Appl. Microbiol. 111, 1327-1336.

49. Jia, X.H., Zhang, C.L., Shi, D.J., Zhuang, M.M., Wang, X., Jia, R., Zhang, Z.Y., Huang, J., Sun, Y.H., Qian, W.Y., 2015. Oral administration of Anabaena-expressed VP28 for both drug and food against white spot syndrome virus in shrimp. J. Appl. Phycol. 28, 1001-1009.

50. Chlamydomonas in the Laboratory. In: The Chlamydomonas Sourcebook 3-Vol set. Elsevier Inc.; 2009. p. 241-302 
3 Figure 1 The pASapI expression vector contains the atpA promoter/5'UTR, a multiple

\section{LIST OF FIGURES}

cloning site for coding sequence of the gene of interest (GOI) insertion, the $r b c L$ 3'UTR and the essential photosynthesis gene $p s b H$ which serves as a selectable marker. C. reinhardtii recipient strain TN72 is non-photosynthetic because $p s b H$ has been disrupted using the aadA gene that confers spectinomycin resistance. Following transformation, two homologous recombination events result in the replacement of $p s b H:: a a d A$ with a functional $p s b H$ and with the expression cassette harboring the GOI.

Figure 2 Nucleotide sequence alignment of the original $v p 28$ sequence (top sequence) and the codon optimized $v p 28$ ( $v p 28-o p$, bottom sequence). The black shading indicates identical nucleotides. The numbers on the right indicated the position of nucleotides.

Figure 3 PCR confirmation of homoplasmy of $v p 28-o p$ and $v p 28$ transformant lines. A three-primer PCR strategy (see Fig 1) was used to confirm the correct integration of the expression cassette into the chloroplast genome of the phototrophic transformants. Heteroplasmic transformants harboring both transformed and untransformed copies of the genome would be expected to produce both a $1.2 \mathrm{~kb}$ and a $1.0 \mathrm{~kb}$ PCR product. The absence of any detectable $1.0 \mathrm{~kb}$ band suggests that all transformant lines are homoplasmic. $\mathrm{M}=$ DNA ladder of size markers, Lane 1-5: transformants with vp28-op, Lane 6-11= transformants with $v p 28$.

Figure 4 Growth on solid media to test for spectinomycin resistance in the different transformant lines. Cultures of: (A) TNVP28-op (OP1-5), (B) TNVP28 (CV1-6) and (C) TNVP28-op (OP), TNVP28 (CV), TN72-emp (CP) and the TN72 recipient strain were grown on TAP medium (left) and TAP containing $100 \mu \mathrm{g} / \mathrm{ml}$ spectinomycin (right). After 1 week, no significant growth of the transformant line is seen on TAP medium containing spectinomycin. Only the untransformed TN72 could grow on the selective medium.

Figure 5 Western blot analysis of total lysate from selected C. reinhardtii transformant lines using a polyclonal anti-VP28 antibody. A positive control (lane P) gives a distinct band at $\sim 28 \mathrm{kDa}$ and a similar sized band is seen in the recombinant VP28-op extracts (OP1 and OP2). A non-specific band at $\sim 35 \mathrm{kDa}$ is seen in all algal samples including TN72 recipient 
and probably represents an endogenous protein that cross-reacts with the antibody (black arrow). (Lane $\mathrm{M}=$ protein marker; $\mathrm{OP} 1$ and $\mathrm{OP} 2=\mathrm{TNVP} 28$-op transformants, $\mathrm{CV}=$ TNVP28, CP = TN-empty, TN72 $=$ TN72 recipient, $\mathrm{P}=$ lysate from $E$. coli expressing recombinant VP28 as a positive control).

Figure 6 Western blot analysis of the His-tagged VP28 from C. reinhardtii transformant (TNVP28-op) affinity purified using a Ni-NTA column. The $28 \mathrm{kDa}$ protein is detected by the anti-VP28 antibody in all eluted fractions. (Lane M, protein marker; lane FT = flowthrough protein fraction that did not bind to the column containing the endogenous $35 \mathrm{kDa}$ protein; lane $\mathrm{W}=$ last wash fraction; lane E1 - E2 = first eluted fraction with 100 and 250 $\mathrm{mM}$ imidazole; lane E3 - E5 = eluted fraction with $500 \mathrm{mM}$ imidazole; lane $\mathrm{C}=$ extracted proteins from E. coli expressing VP28 as a positive control).

Figure 7 C. reinhardtii cells incubated in TAP medium at different $\mathrm{pH}$ 's for 1, 3 and 5 hours to examine the acid tolerance.

Figure 8 C. reinhardtii cells incubating in different bile salt concentrations for 30, 60 and $90 \mathrm{~min}$ to examine the bile salt tolerance.

6 Figure 9 Cumulative mortality rates of shrimp against WSSV from the groups orally 7 immunostimulated with $C$. reinhardtii strain TNVP28-op, TNVP28 and TN72-emp. As a 8 positive control, shrimps were fed only commercial feed. Shrimps were challenged with 9 WSSV 10 days' post-immunization and cumulative mortalities were recorded 8 days after 60 challenge. A negative control group were not challenged with the WSSV 
61 FIGURE 1
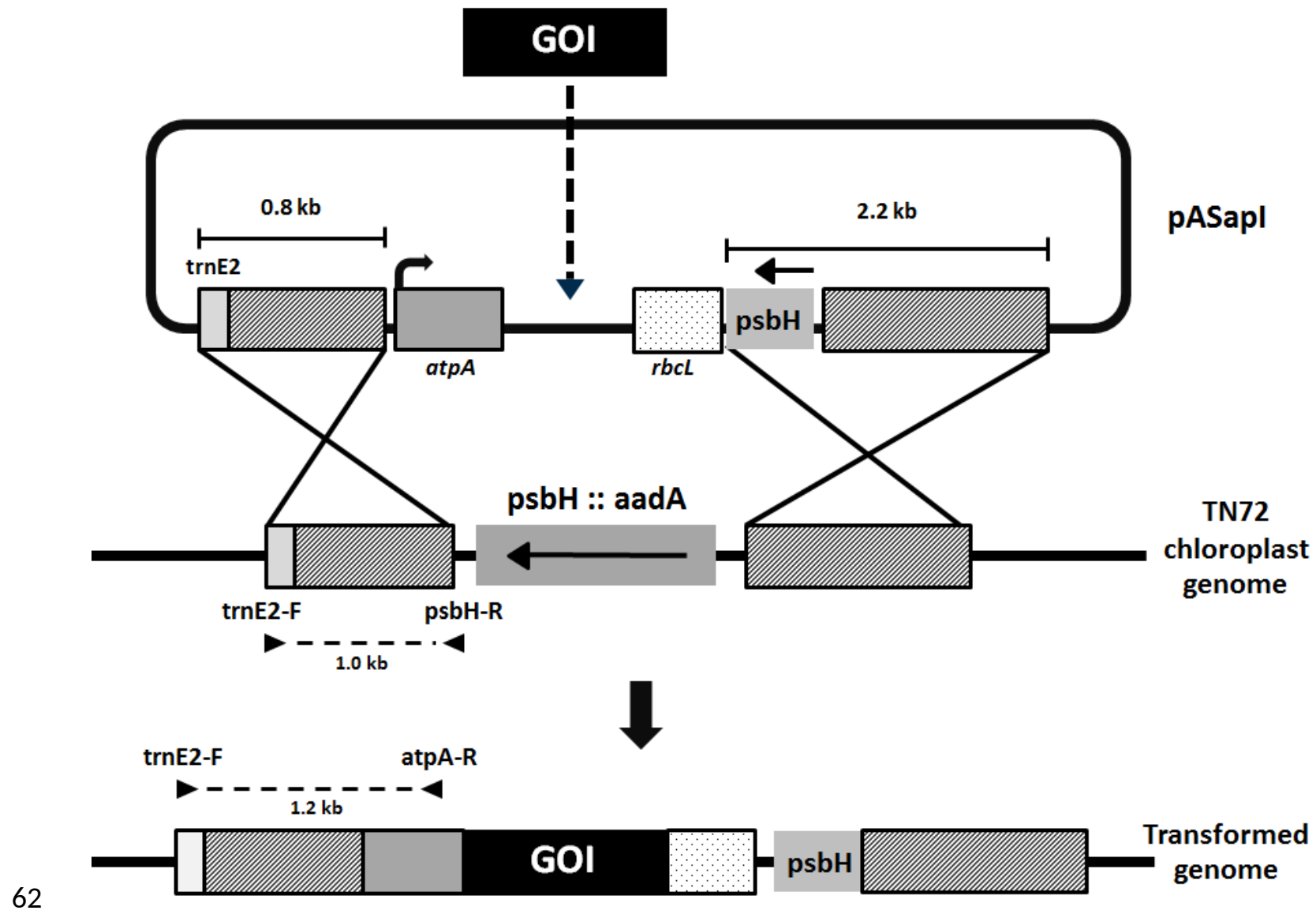
FIGURE 2

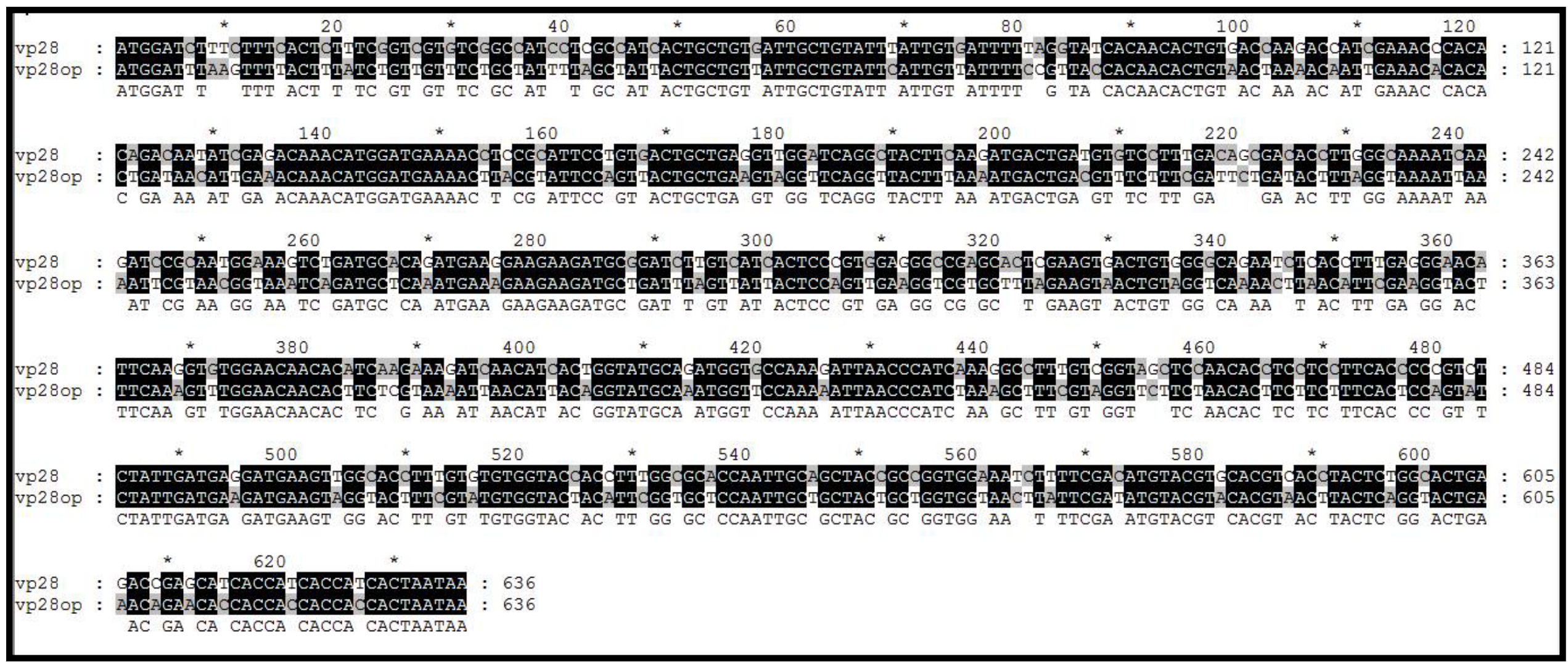


65 FIGURE 3

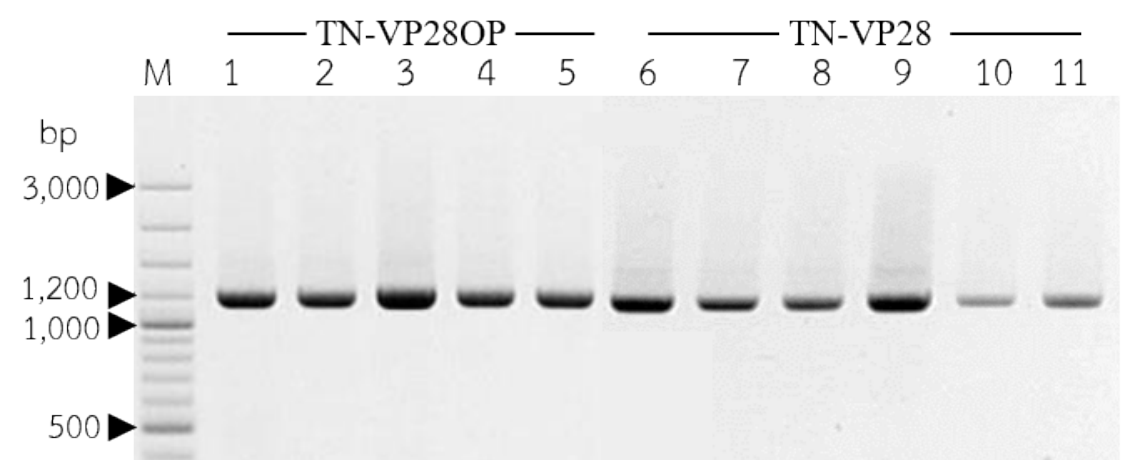


67 FIGURE 4

68

69

70

71

72

73

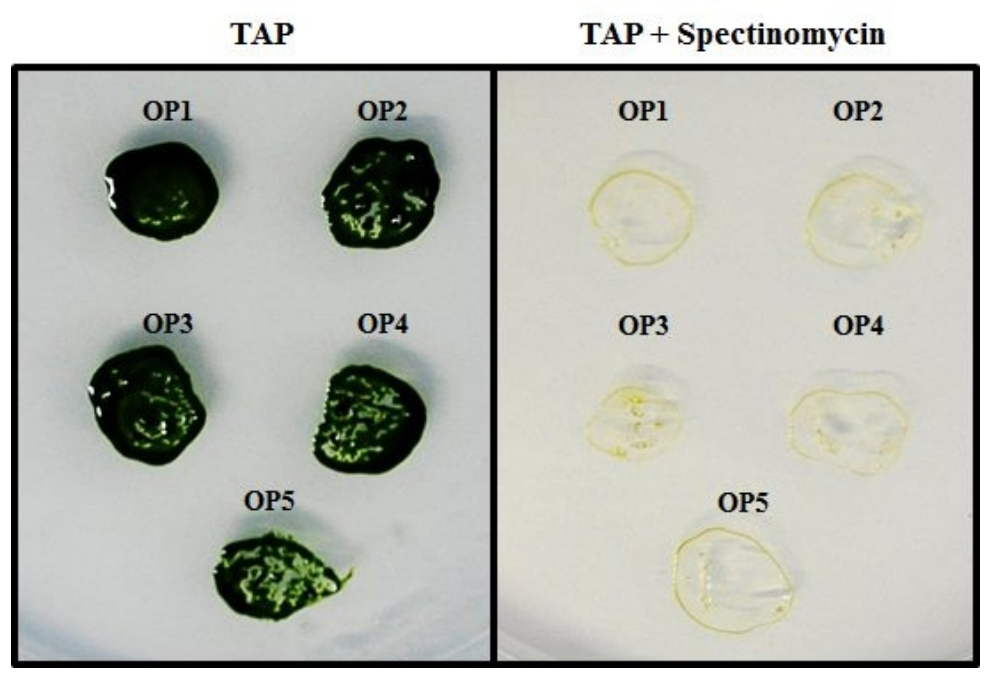

(A)

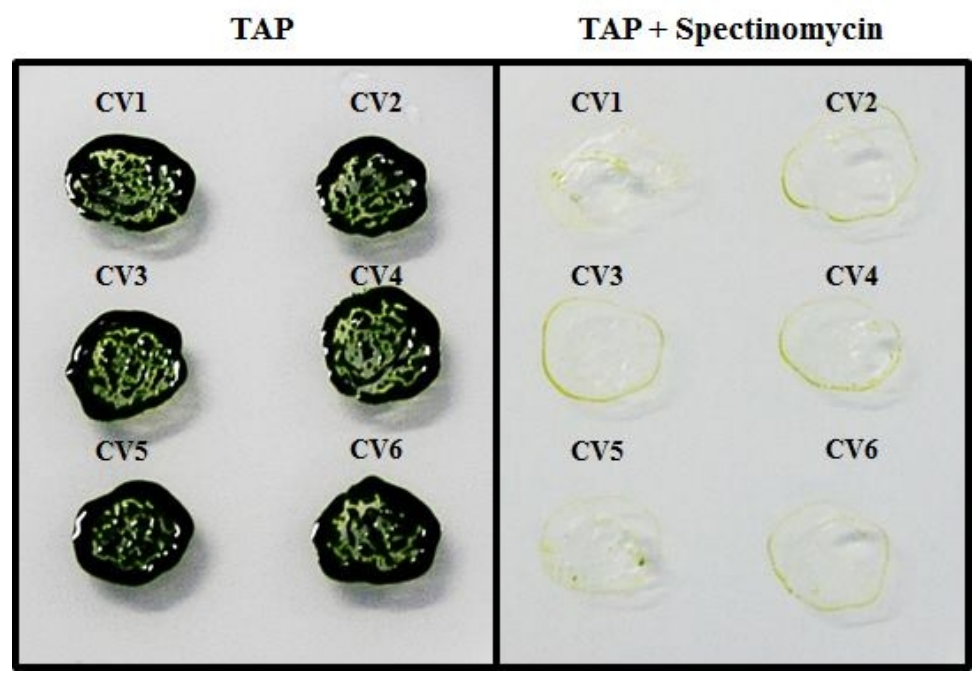

(B)

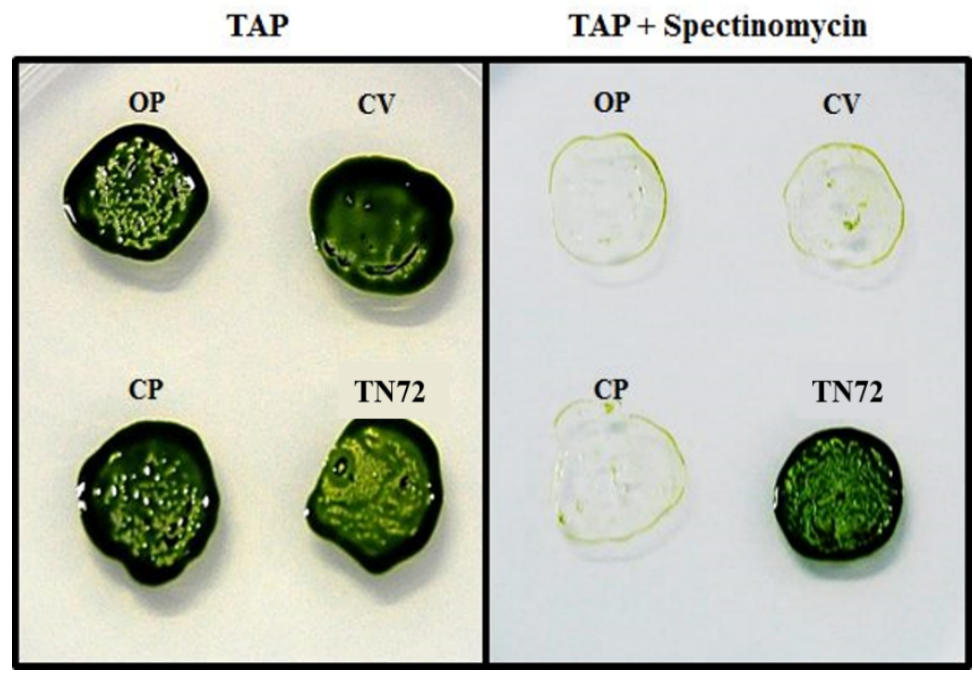

(C) 
74 FIGURE 5

75

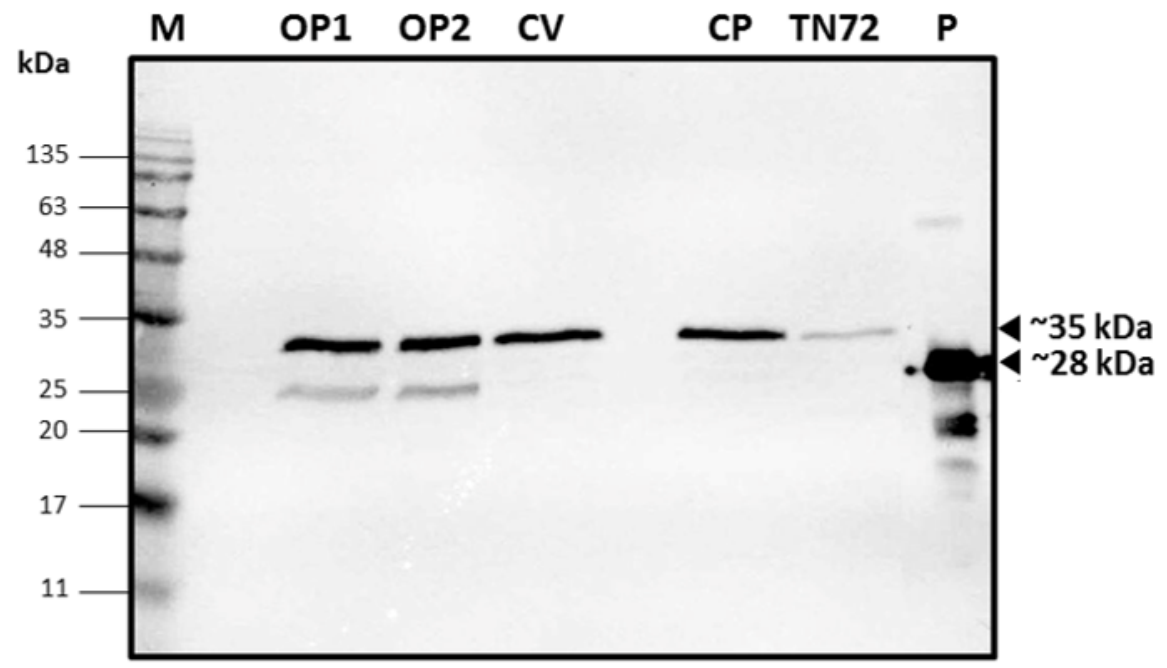

76 
79 FIGURE 6

80

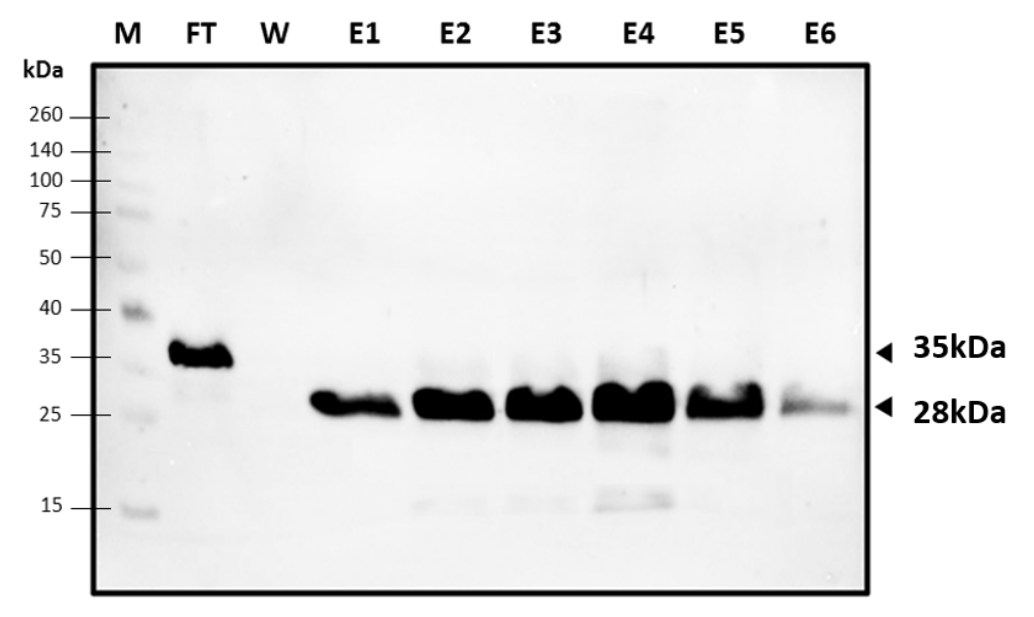


FIGURE 7

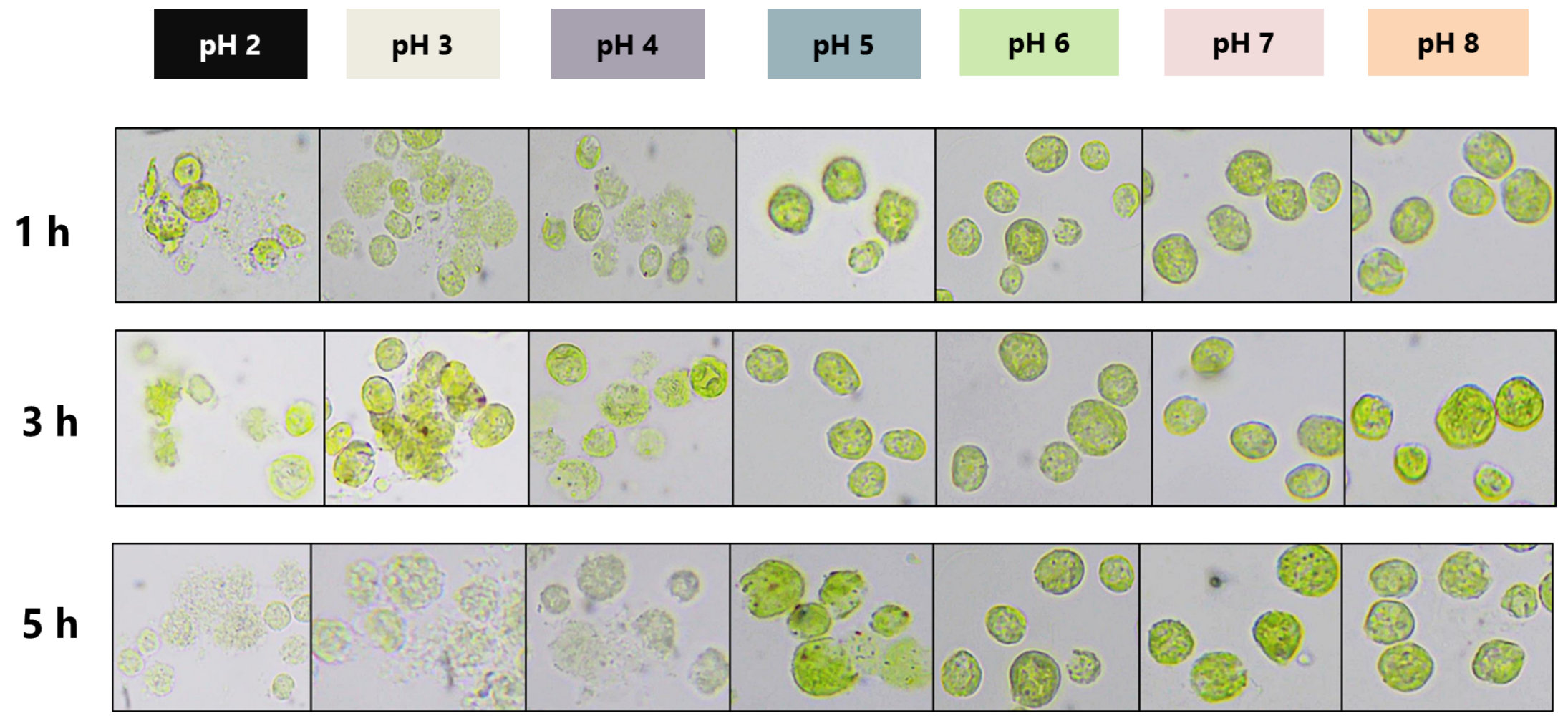


FIGURE 8

86

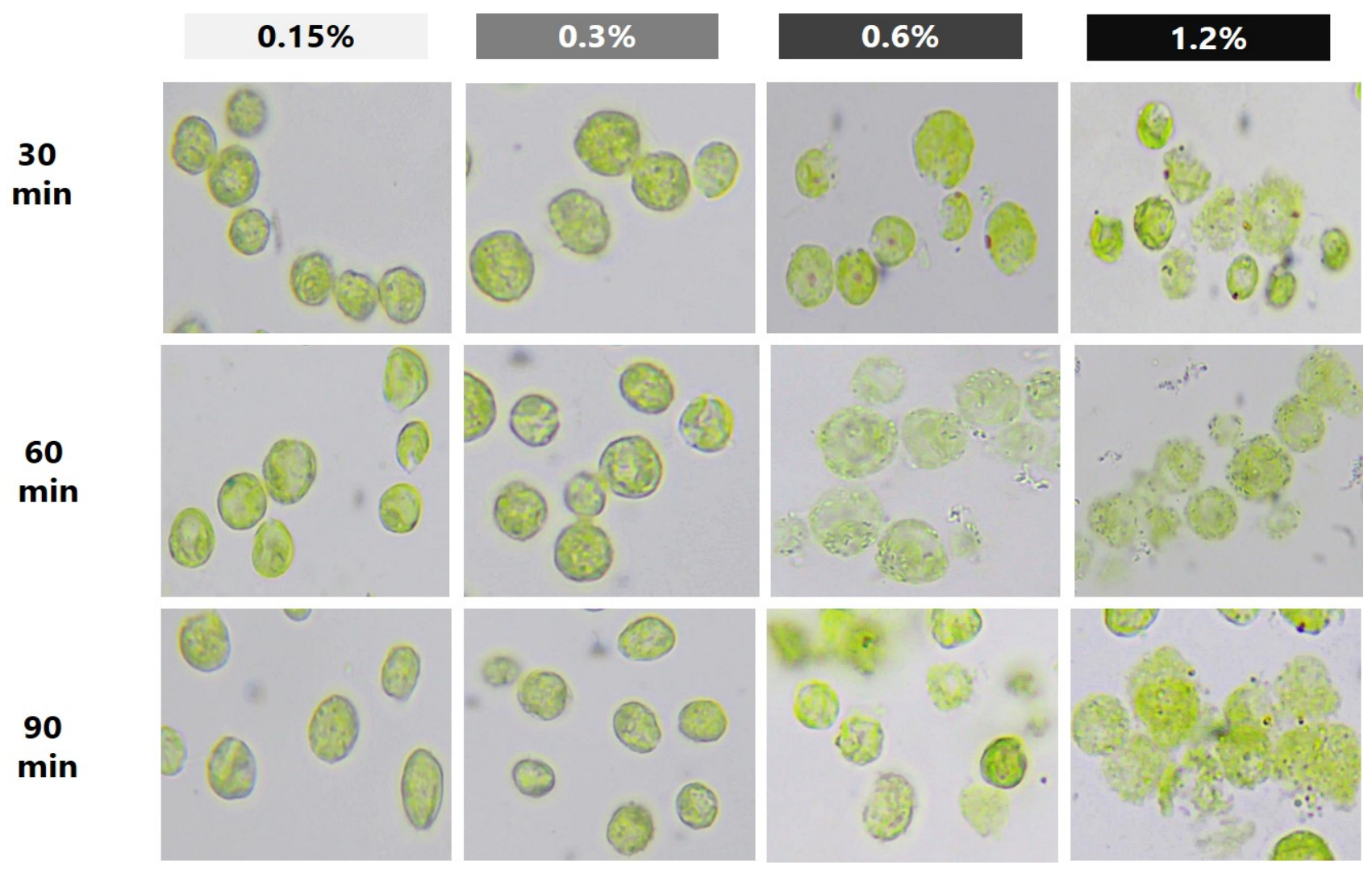




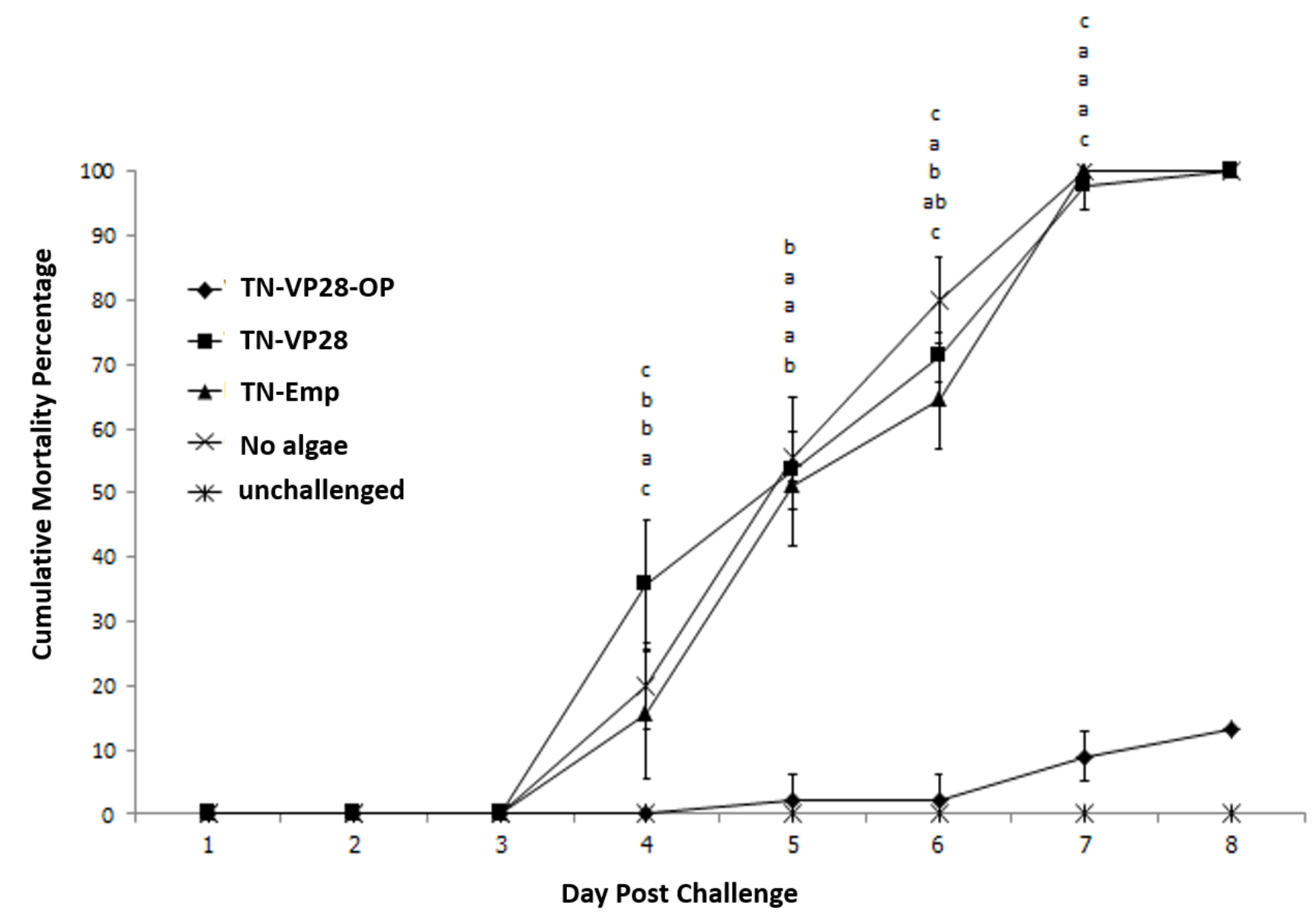

